\title{
Excited states and reduced transition probabilities in ${ }^{168} \mathrm{Os}$
}

T. Grahn,,${ }^{1,2,{ }^{*}}$ S. Stolze,${ }^{2}$ D. T. Joss, ${ }^{1}$ R. D. Page,${ }^{1}$ B. Sayğ $1,{ }^{1, \dagger}$ D. O’Donnell, ${ }^{1}$ M. Akmali, ${ }^{1}$ K. Andgren,${ }^{3}$ L. Bianco, ${ }^{1}$ D. M. Cullen, ${ }^{4}$ A. Dewald, ${ }^{5}$ P. T. Greenlees, ${ }^{2}$ K. Heyde, ${ }^{6}$ H. Iwasaki, ${ }^{5}$ U. Jakobsson, ${ }^{2}$ P. Jones, ${ }^{2}$ D. S. Judson, ${ }^{1}$ R. Julin, ${ }^{2}$ S. Juutinen, ${ }^{2}$ S. Ketelhut, ${ }^{2}$ M. Leino, ${ }^{2}$ N. Lumley, ${ }^{4}$ P. J. R. Mason, ${ }^{4,7}$ O. Möller, ${ }^{8}$ K. Nomura,${ }^{5,9}$ M. Nyman, ${ }^{2}$ A. Petts, ${ }^{1}$ P. Peura, ${ }^{2}$ N. Pietralla, ${ }^{8}$ Th. Pissulla, ${ }^{5}$ P. Rahkila, ${ }^{2}$ P. J. Sapple, ${ }^{1}$ J. Sarén, ${ }^{2}$ C. Scholey, ${ }^{2}$ J. Simpson, ${ }^{7}$ J. Sorri, ${ }^{2}$ P. D. Stevenson, ${ }^{10}$ J. Uusitalo, ${ }^{2}$ H. V. Watkins, ${ }^{1}$ and J. L. Wood ${ }^{11}$

${ }^{1}$ Department of Physics, Oliver Lodge Laboratory, University of Liverpool, Liverpool L69 7ZE, United Kingdom

${ }^{2}$ University of Jyvaskyla, Department of Physics, P.O. Box 35, FI-40014 University of Jyvaskyla, Finland

${ }^{3}$ Department of Physics, Royal Institute of Technology, SE-10691 Stockholm, Sweden

${ }^{4}$ Department of Physics and Astronomy, University of Manchester, Manchester M13 9PL, United Kingdom

${ }^{5}$ Institut für Kernphysik, Universität zu Köln, 50937 Köln, Germany

${ }^{6}$ Department of Physics and Astronomy, Ghent University, Proeftuinstraat 86, B-9000 Gent, Belgium

${ }^{7}$ STFC Daresbury Laboratory, Daresbury, Warrington WA4 4AD, United Kingdom

${ }^{8}$ Institut für Kernphysik, TU Darmstadt, 64289 Darmstadt, Germany

${ }^{9}$ Department of Physics, University of Tokyo, Hongo, Bunkyo-ku, Tokyo 113-003, Japan

${ }^{10}$ Department of Physics, University of Surrey, Guildford, Surrey GU2 7XH, United Kingdom

${ }^{11}$ School of Physics, Georgia Institute of Technology, Atlanta, Georgia 30332-0430, USA

(Received 19 July 2016; revised manuscript received 2 September 2016; published 28 October 2016)

\begin{abstract}
The level scheme of the neutron-deficient nuclide ${ }^{168}$ Os has been extended and mean lifetimes of excited states have been measured by the recoil distance Doppler-shift method using the JUROGAM $\gamma$-ray spectrometer in conjunction with the IKP Köln plunger device. The ${ }^{168}$ Os $\gamma$ rays were measured in delayed coincidence with recoiling fusion-evaporation residues detected at the focal plane of the RITU gas-filled separator. The ratio of reduced transition probabilities $B\left(E 2 ; 4_{1}^{+} \rightarrow 2_{1}^{+}\right) / B\left(E 2 ; 2_{1}^{+} \rightarrow 0_{1}^{+}\right)$is measured to be $0.34(18)$, which is very unusual for collective band structures and cannot be reproduced by interacting boson model (IBM-2) calculations based on the $\mathrm{SkM}^{*}$ energy-density functional.
\end{abstract}

DOI: 10.1103/PhysRevC.94.044327

\section{INTRODUCTION}

The emergence of collective behavior in atomic nuclei outside closed shell configurations represents one of the most important paradigms in the description of many-body quantum mechanical systems $[1,2]$. The systematics of excited states reveal fundamental information about the evolution of nuclear structure [3]. The largest range of isotopes between consecutive magic numbers that can be synthesized and studied in their excited states is found within the $82 \leqslant N \leqslant 126$ shell. The longest continuous chain with known excited states is for osmium, which spans 37 isotopes from ${ }^{162} \mathrm{Os}(N=86)$ [4] to ${ }^{198}$ Os $(N=122)$ [5]. The Os excitation energy systematics $E\left(4_{1}^{+}\right) / E\left(2_{1}^{+}\right)$show the development from single-particle excitations near the $N=82$ closed shell [4,6], through soft triaxial rotors $(N \sim 92)[7]$ and shape coexistence $(N=96)$ $[8,9]$ towards well-deformed prolate shapes near the midshell at $N=104$. Beyond $N=104$ collectivity decreases towards $N=126$ due to the diminishing valence space $[5,10,11]$; see Fig. 1(a).

Reduced transition probabilities, particularly $B(E 2)$ values, serve as a more sensitive experimental probe for analyzing collectivity as a function of nucleon number. The $B(E 2)$ values usually increase with spin for low-lying states within a

\footnotetext{
*tuomas.grahn@jyu.fi

${ }^{\dagger}$ Also at Department of Physics, Faculty of Science, Ege University, Bornova, Izmir 35100, Turkey.
}

rotational band [2]. A consequence of this feature is that the $B\left(E 2 ; 4_{1}^{+} \rightarrow 2_{1}^{+}\right) / B\left(E 2 ; 2_{1}^{+} \rightarrow 0_{1}^{+}\right)$ratio (hereafter known as $B_{4 / 2}$ ) is larger than unity for collective excitations: 1.43 and 2.0 for an ideal rotor and vibrator, respectively. However, in nuclei near closed shells where single-particle motion dominates the nuclear structure, this increasing trend of the $B(E 2)$ values can be reversed [12]. Ten nuclei with $B_{4 / 2}$ ratios less than unity were identified in a survey of reduced transition probability ratios [13]. Although some of the cases have been remeasured using complementary experimental techniques and the anomaly rectified (e.g. ${ }^{98} \mathrm{Ru}$ and ${ }^{180} \mathrm{Pt}$ ) [14], the anomaly persists in several other nuclei, such as ${ }^{114} \mathrm{Xe}$ and ${ }^{114} \mathrm{Te}[15,16]$. Further anomalous cases have since been reported, including ${ }^{72,74} \mathrm{Zn}$ [17] and ${ }^{48,50} \mathrm{Cr}$ [18].

This paper reports an extension to the level scheme in ${ }^{168} \mathrm{Os}$ and the measurement of mean lifetimes of excited states using the recoil distance Doppler-shift (RDDS) method. The anomalously low value for the $B_{4 / 2}$ ratio deduced from the measurements cannot be reproduced by interacting boson model (IBM-2) calculations based on the SkM* energy-density functional.

\section{EXPERIMENTAL DETAILS}

Excited states of ${ }^{168}$ Os were populated in the fusionevaporation reactions in experiments performed at the Accelerator Laboratory of the University of Jyväskylä listed in Table I. In each experiment $\gamma$ rays were detected with the JUROGAM $\gamma$-ray spectrometer consisting of 43 Eurogam 


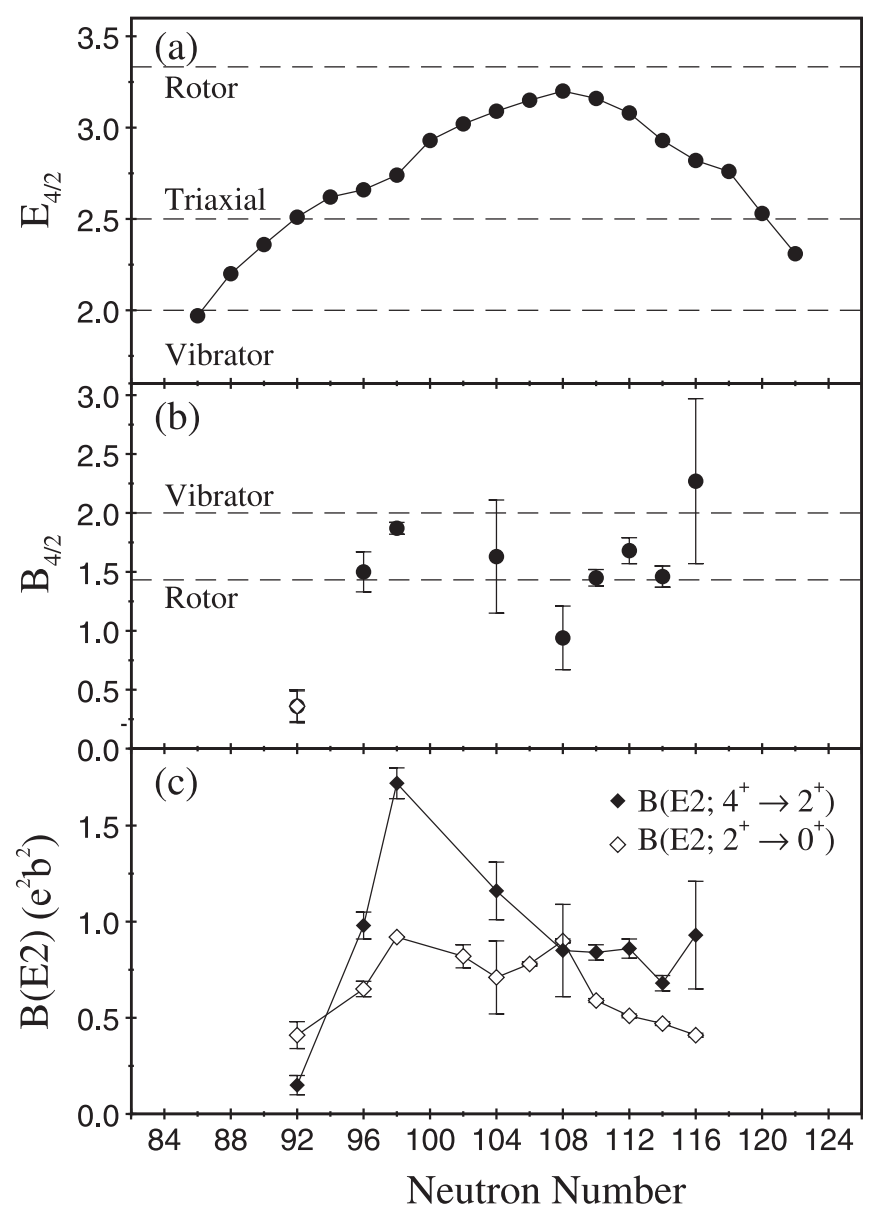

FIG. 1. (a) Ratios of the $4^{+}$to $2^{+}$state excitation energies for the even-mass Os isotopes. (b) Ratios of the reduced transition probabilities $\left(B_{4 / 2}\right)$ measured for the even-mass Os isotopes. (c) Measured reduced transition probabilities $B(E 2)$ for the $4^{+} \rightarrow 2^{+}$ (filled diamonds) and $2^{+} \rightarrow 0^{+}$(open diamonds) transitions. The measured values for ${ }^{168}$ Os correspond to neutron number $N=92$. Data for the heavier isotopes were obtained from Ref. [19].

Phase I type escape-suppressed spectrometers [20] arranged in six angular groups with respect to the beam direction. Evaporation residues recoiling from the target were separated in flight from unreacted beam and fission fragments according to their magnetic rigidity by the RITU gas-filled separator [21] before being implanted into the GREAT spectrometer [22] located at the focal plane. Recoiling fusion residues were distinguished from background events by their energy loss in the GREAT multiwire proportional counter and, in conjunction with the GREAT double-sided silicon strip detectors (DSSDs), by their time-of-flight characteristics. These data were recorded using the Total Data Readout data acquisition system [23] and analyzed offline using the GRAIN [24] and RADWARE software packages [25]. All $\gamma$-ray data were recorded in delayed coincidence with fusion-evaporation residues implanted into the GREAT spectrometer.

In experiments 1 and 2 a high-fold coincidence analysis was performed leading to a significant extension of the ${ }^{168} \mathrm{Os}$ level scheme. In experiment 3 the standard JUROGAM target chamber was replaced by the IKP Köln plunger device [26], in which the distance between the target and a degrader foil was varied. This allowed lifetimes to be measured for excited states using the RDDS method. The stretched self-supporting ${ }^{92}$ Mo target had a thickness of $1 \mathrm{mg} / \mathrm{cm}^{2}$ while the degrader was a $1 \mathrm{mg} / \mathrm{cm}^{2}$ thick $\mathrm{Mg}$ foil. The reaction provided a recoil velocity of $v / c=3.8 \%$ and $2.8 \%$ before and after the degrader, respectively.

\section{RESULTS}

\section{A. $\gamma \gamma \gamma$ coincidence analysis}

A total of $9.3 \times 10^{6}$ recoil- $\gamma^{n}(n \geqslant 3)$ coincidences were recorded in the combined data from experiments 1 and 2 and sorted into an $E_{\gamma 1}-E_{\gamma_{2}}-E_{\gamma_{3}}$ coincidence cube. The level scheme for ${ }^{168}$ Os was constructed on the basis of relative $\gamma$-ray intensities and coincidence relationships. The deduced level scheme for ${ }^{168}$ Os is displayed in Fig. 2 and the properties of $\gamma$ rays assigned to this nucleus are recorded in Table II.

$\gamma$-ray transitions in ${ }^{168} \mathrm{Os}$ were first identified using the recoil-decay tagging technique and a high spin level scheme was established [7]. In the present work the aligned $v i_{13 / 2}^{2}$ band (band 2) has been extended from the $\left(18^{+}\right)$state by two transitions. The negative-parity bands (bands 3 and 4) are similarly extended to the $\left(21^{-}\right)$and $\left(20^{-}\right)$states by two and one transitions, respectively. Figure 3 shows typical double-gated spectra highlighting these extensions of the ${ }^{168} \mathrm{Os}$ level scheme. Band 2 in Fig. 2, is fed by a $643 \mathrm{keV}$ transition that is unresolved from the $642 \mathrm{keV}\left(6_{1}^{+} \rightarrow 4_{1}^{+}\right)$transition in the ground-state band. Figure 3(a) shows $\gamma$ rays in coincidence with both 642 and $643 \mathrm{keV}$ transitions and supports the placement of the $643 \mathrm{keV} \gamma$-ray transition feeding band 2 . Figures 3(b) and 3(c) show typical coincidence spectra used to extend bands 3 and 4 , respectively.

The $\left(12^{+}\right)$state in band 2 was known previously to have discrete $\gamma$-ray branches to the ground-state band and the negative-parity side bands, bands 3 and 4 . Two further depopulation paths from the $\left(12^{+}\right)$state have been established;

TABLE I. Summary of reactions employed in the present work. The data from experiments 1 and 2 were used for the $\gamma$-ray coincidence analysis, while those from experiment 3 were used for the lifetime measurements.

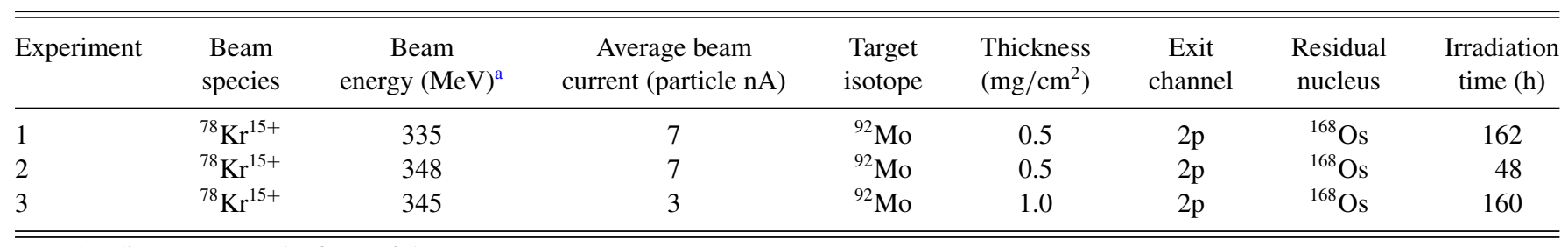

${ }^{\mathrm{a} B o m b a r d i n g ~ e n e r g y ~ a t ~ t h e ~ f r o n t ~ o f ~ t h e ~ t a r g e t . ~}$ 


\section{Band $1 \quad$ Band 2}

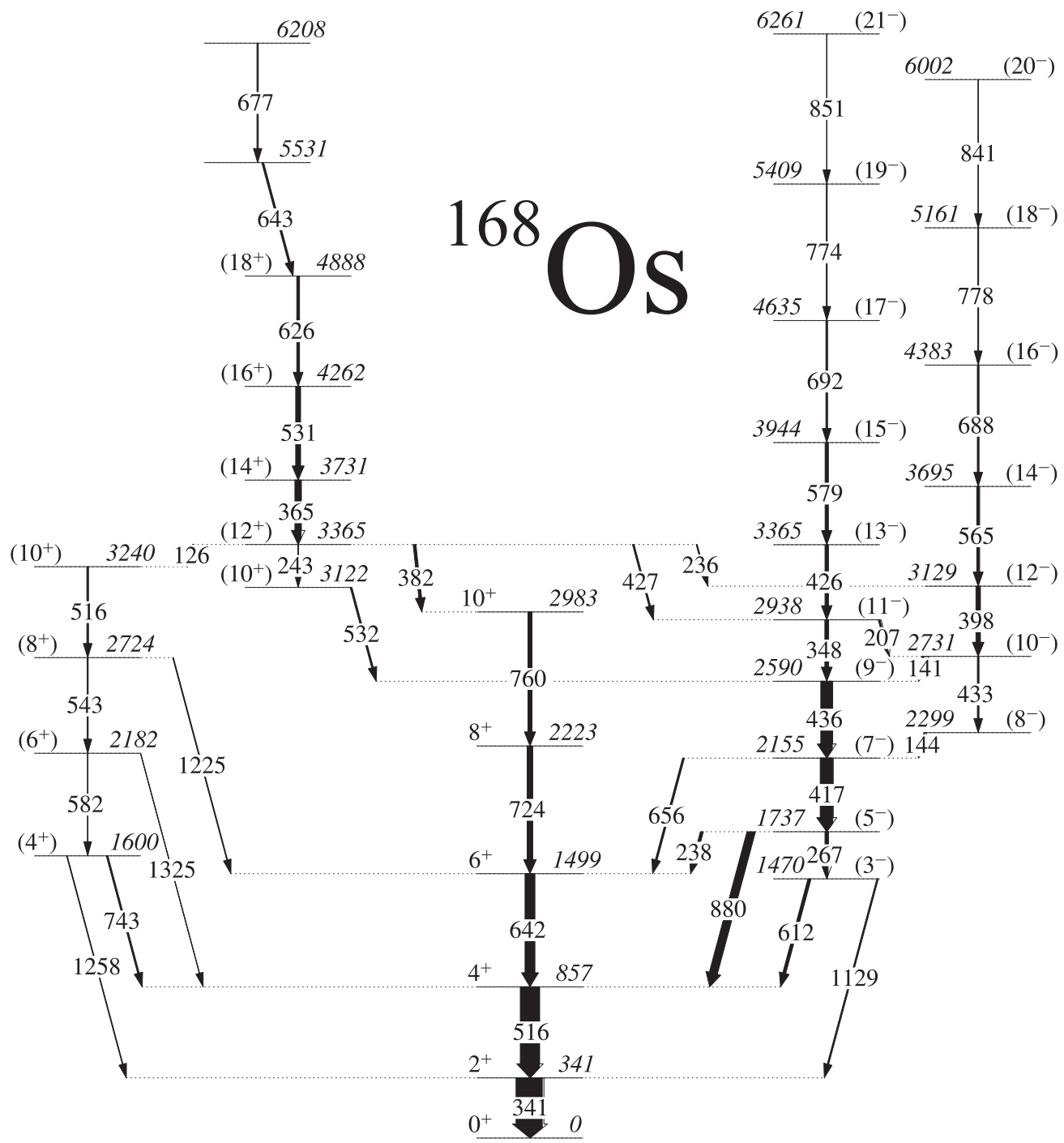

\section{Band 3 Band 4}

\section{Ground-State Band}

FIG. 2. Level scheme deduced for ${ }^{168} \mathrm{Os}$. The transition energies are given in $\mathrm{keV}$ and their relative intensities are proportional to the widths of the arrows. Excited states are labeled with their excitation energies relative to the ground state and their spin and parity assignments. Parentheses indicate tentative assignments.

see Fig. 2. The $243 \mathrm{keV}$ and the $532 \mathrm{keV}$ transitions feed band 3 , while the $126 \mathrm{keV}$ transition feeds a new band structure, band 1. It was not possible to measure the multipolarities of the transitions in the new band. However, a positive-parity even-spin structure is inferred from the nature of the $\gamma$-ray transition branches to the low-spin states of the ground-state band. The spectra highlighting these decay paths are shown in Figs. 4 and 5. The $591 \mathrm{keV}$ transition in Fig. 4(b) appears to feed the level at $3122 \mathrm{keV}$.

\section{B. RDDS lifetime measurements}

Recoil-correlated $\gamma$-ray coincidences were recorded at 13 different target-to-degrader distances of the plunger device for $\sim 12$ hours each. The experiment was optimized for the lifetime measurements of the $2^{+}$and $4^{+}$states; i.e., the distances were chosen to span a region of sensitivity where the relative intensities of the fully shifted and degraded components of the depopulating transitions for these states varied; see Fig. 6. The recoil-gated $\gamma$-ray coincidences were analyzed in order to eliminate the influence of unobserved feeding transitions on the lifetimes under investigation [27].

Sufficient $\gamma$-ray data were collected with JUROGAM at the detector angles of $158^{\circ}$ ( 5 detectors) and $134^{\circ}$ (10 detectors) in coincidence with the $\gamma$ rays recorded with all the detectors to allow the measurements of the $2^{+}$and $4^{+}$state lifetimes using the differential decay curve method (DDCM) [28]. In the DDCM mean lifetimes are obtained from the relative 
TABLE II. Measured properties of $\gamma$-ray transitions assigned to ${ }^{168}$ Os. Energies are accurate to $\pm 0.5 \mathrm{keV}$ for the strong $\left(I_{\gamma}>10 \%\right)$ transitions rising to $\pm 2.0 \mathrm{keV}$ for the weaker transitions.

\begin{tabular}{|c|c|c|c|c|c|}
\hline $\begin{array}{l}E_{\gamma} \\
(\mathrm{keV})\end{array}$ & $\begin{array}{c}I_{\gamma} \\
(\%)\end{array}$ & $\begin{array}{c}E_{\mathrm{i}} \\
(\mathrm{keV})\end{array}$ & $I_{\mathrm{i}}$ & & $I_{\mathrm{f}}$ \\
\hline 125.8 & $1.97(15)$ & 3365 & $\left(12^{+}\right)$ & $\rightarrow$ & $\left(10^{+}\right)$ \\
\hline 140.5 & $7.6(5)$ & 2731 & $\left(10^{-}\right)$ & $\rightarrow$ & $\left(9^{-}\right)$ \\
\hline 144.5 & $3.3(3)$ & 2299 & $\left(8^{-}\right)$ & $\rightarrow$ & $\left(7^{-}\right)$ \\
\hline 207.0 & $6.1(4)$ & 2938 & $\left(11^{-}\right)$ & $\rightarrow$ & $\left(10^{-}\right)$ \\
\hline 236.1 & $3.8(3)$ & 3365 & $\left(12^{+}\right)$ & $\rightarrow$ & $\left(12^{-}\right)$ \\
\hline 237.6 & $10.6(7)$ & 1737 & $\left(5^{-}\right)$ & $\rightarrow$ & $6^{+}$ \\
\hline 243.3 & $2.31(18)$ & 3365 & $\left(12^{+}\right)$ & $\rightarrow$ & $\left(10^{+}\right)$ \\
\hline 267.0 & $12.0(8)$ & 1737 & $\left(5^{-}\right)$ & $\rightarrow$ & $\left(3^{-}\right)$ \\
\hline 341.1 & $100(7)$ & 341 & $2^{+}$ & $\rightarrow$ & $0^{+}$ \\
\hline 348.4 & $13.6(9)$ & 2938 & $\left(11^{-}\right)$ & $\rightarrow$ & $\left(9^{-}\right)$ \\
\hline 365.4 & $23.5(15)$ & 3731 & $\left(14^{+}\right)$ & $\rightarrow$ & $\left(12^{+}\right)$ \\
\hline 382.3 & $10.1(7)$ & 3365 & $\left(12^{+}\right)$ & $\rightarrow$ & $10^{+}$ \\
\hline 398.3 & $16.5(10)$ & 3129 & $\left(12^{-}\right)$ & $\rightarrow$ & $\left(10^{-}\right)$ \\
\hline 417.3 & $48(3)$ & 2155 & $\left(7^{-}\right)$ & $\rightarrow$ & $\left(5^{-}\right)$ \\
\hline 426.0 & $12.7(9)$ & 3365 & $\left(13^{-}\right)$ & $\rightarrow$ & $\left(11^{-}\right)$ \\
\hline 426.8 & $5.9(5)$ & 3365 & $\left(12^{+}\right)$ & $\rightarrow$ & $\left(11^{-}\right)$ \\
\hline 432.7 & $6.0(5)$ & 2731 & $\left(10^{-}\right)$ & $\rightarrow$ & $\left(8^{-}\right)$ \\
\hline 435.5 & $44(3)$ & 2590 & $\left(9^{-}\right)$ & $\rightarrow$ & $\left(7^{-}\right)$ \\
\hline 515.5 & $6.2(6)$ & 3240 & $\left(10^{+}\right)$ & $\rightarrow$ & $\left(8^{+}\right)$ \\
\hline 516.3 & $72(5)$ & 857 & $4^{+}$ & $\rightarrow$ & $2^{+}$ \\
\hline 531.1 & $18.3(12)$ & 4262 & $\left(16^{+}\right)$ & $\rightarrow$ & $\left(14^{+}\right)$ \\
\hline 531.9 & $6.3(6)$ & 3122 & $\left(10^{+}\right)$ & $\rightarrow$ & $\left(9^{-}\right)$ \\
\hline 542.6 & $3.8(4)$ & 2724 & $\left(8^{+}\right)$ & $\rightarrow$ & $\left(6^{+}\right)$ \\
\hline 565.5 & $10.8(7)$ & 3695 & $\left(14^{-}\right)$ & $\rightarrow$ & $\left(12^{-}\right)$ \\
\hline 579.1 & $12.2(8)$ & 3944 & $\left(15^{-}\right)$ & $\rightarrow$ & $\left(13^{-}\right)$ \\
\hline 582.0 & $2.2(3)$ & 2182 & $\left(6^{+}\right)$ & $\rightarrow$ & $\left(4^{+}\right)$ \\
\hline 590.8 & $4.4(4)$ & 3713 & & $\rightarrow$ & $\left(10^{+}\right)$ \\
\hline 612.2 & $11.5(8)$ & 1470 & $\left(3^{-}\right)$ & $\rightarrow$ & $4^{+}$ \\
\hline 625.8 & $10.4(7)$ & 4888 & $\left(18^{+}\right.$ & $\rightarrow$ & $\left(16^{+}\right)$ \\
\hline 641.8 & $37.2(24)$ & 1499 & $6^{+}$ & $\rightarrow$ & $4^{+}$ \\
\hline 643.1 & $6.8(5)$ & 5531 & $\left(20^{+}\right)$ & $\rightarrow$ & $\left(18^{+}\right)$ \\
\hline 656.3 & $6.7(5)$ & 2155 & $\left(7^{-}\right)$ & $\rightarrow$ & $6^{+}$ \\
\hline 677.3 & $4.8(3)$ & 6208 & $\left(22^{+}\right)$ & $\rightarrow$ & $\left(20^{+}\right)$ \\
\hline 688.1 & $6.5(5)$ & 4383 & $\left(16^{-}\right)$ & $\rightarrow$ & $\left(14^{-}\right)$ \\
\hline 691.9 & $6.4(5)$ & 4635 & $\left(17^{-}\right)$ & $\rightarrow$ & $\left(15^{-}\right)$ \\
\hline 724.1 & 21.1(14) & 2223 & $8^{+}$ & $\rightarrow$ & $6^{+}$ \\
\hline 743.4 & $5.8(6)$ & 1600 & $\left(4^{+}\right)$ & $\rightarrow$ & $4^{+}$ \\
\hline 760.1 & $15.5(10)$ & 2983 & $10^{+}$ & $\rightarrow$ & $8^{+}$ \\
\hline 774.0 & $3.4(3)$ & 5409 & $\left(19^{-}\right)$ & $\rightarrow$ & $\left(17^{-}\right)$ \\
\hline 778.2 & $3.3(3)$ & 5161 & $\left(18^{-}\right)$ & $\rightarrow$ & $\left(16^{-}\right)$ \\
\hline 840.8 & $1.04(15)$ & 6002 & $\left(20^{-}\right)$ & $\rightarrow$ & $\left(18^{-}\right)$ \\
\hline 851.2 & $1.35(16)$ & 6261 & $\left(21^{-}\right)$ & $\rightarrow$ & $\left(19^{-}\right)$ \\
\hline 879.9 & $31.3(20)$ & 1737 & $\left(5^{-}\right)$ & $\rightarrow$ & $4^{+}$ \\
\hline 1128.8 & $5.3(5)$ & 1470 & $\left(3^{-}\right)$ & $\rightarrow$ & $2^{+}$ \\
\hline 1224.8 & $3.0(3)$ & 2724 & $\left(8^{+}\right)$ & $\rightarrow$ & $6^{+}$ \\
\hline 1257.7 & $2.7(5)$ & 1600 & $\left(4^{+}\right)$ & $\rightarrow$ & $\left(4^{+}\right)$ \\
\hline 1325.2 & $1.5(3)$ & 2182 & $\left(6^{+}\right)$ & $\rightarrow$ & $4^{+}$ \\
\hline
\end{tabular}

intensity variation with distance of the fully Doppler-shifted and degraded components of the $\gamma$-ray transitions feeding and depopulating the level of interest through the equation

$$
\tau=\frac{Q_{\text {depop }}^{d}(x)-Q_{\text {feed }}^{d}(x)}{v \frac{d}{d x}\left[Q_{\text {depop }}^{s}(x)\right]},
$$

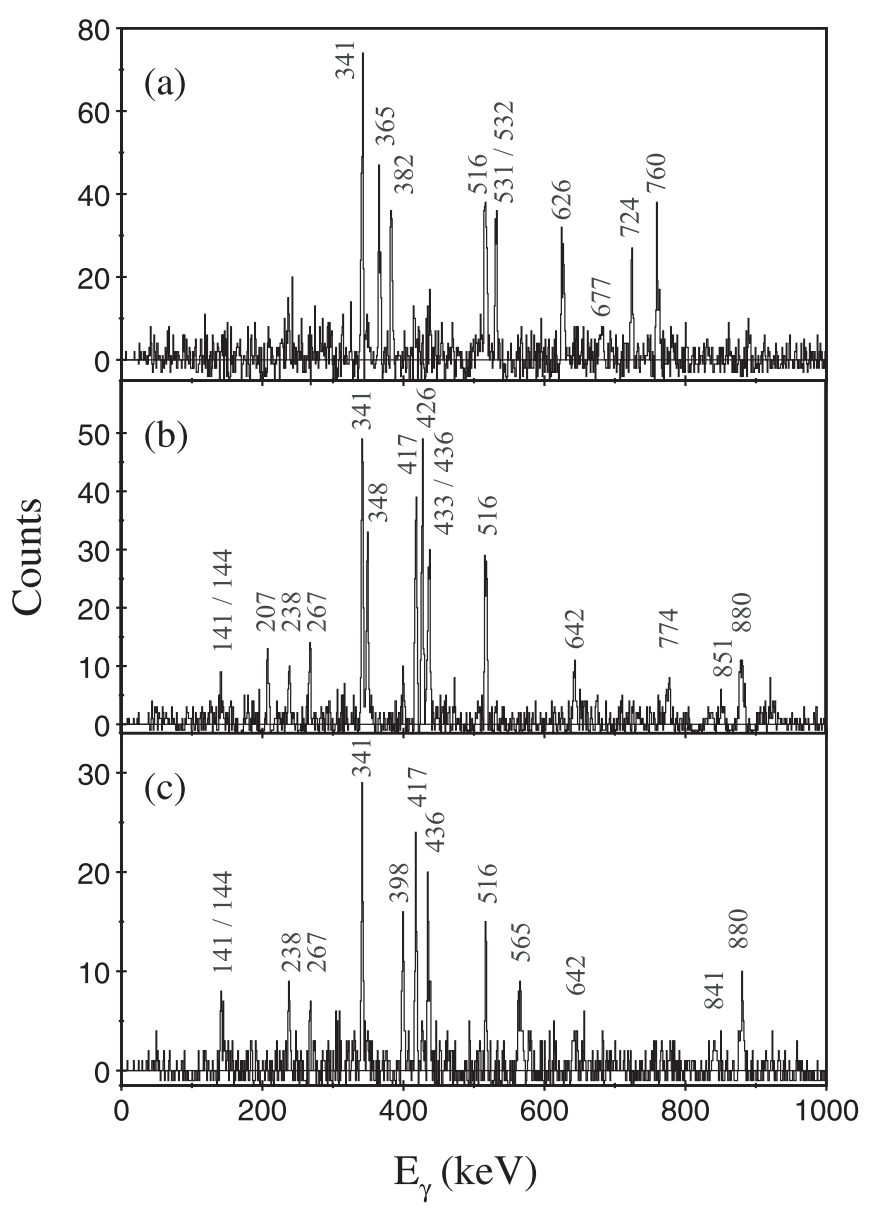

FIG. 3. Double-gated $\gamma$-ray coincidence spectra correlated with a recoil implantation in the GREAT spectrometer. (a) Spectrum showing $\gamma$ rays in coincidence with the 642 and $643 \mathrm{keV}$ transitions. (b) Spectrum showing $\gamma$ rays in coincidence with the 579 and $692 \mathrm{keV}$ transitions in band 3. (c) Spectrum showing $\gamma$ rays in coincidence with the 688 and $778 \mathrm{keV}$ transitions in band 4 .

where $Q_{j}^{i}(x)=I_{j}^{i} /\left(I_{j}^{s}+I_{j}^{d}\right)$ and $I_{j}^{i}(x)$ are the $\gamma$-ray intensities for the shifted $(i=s)$ and degraded $(i=d)$ components measured at the target-to-degrader distance $x$ for the depopulating $(j=$ depop) and feeding $(j=$ feed $)$ transitions, respectively. Therefore, the $\gamma$-ray intensities $I$ recorded with different distances $x$ are normalized by the sum of their fully shifted and degraded components. The final lifetime is an error-weighted average of individual lifetimes [Eq. (1)] obtained at different target-to-degrader distances within the region of sensitivity where the derivative of the decay curve is greater than zero. Lifetime determination for the $2^{+}$and $4^{+}$ states are as shown in Figs. 7 and 8, respectively.

In order to obtain statistically viable $\gamma$-ray spectra, coincidences were demanded between the full line shape of the $516 \mathrm{keV}\left(4^{+} \rightarrow 2^{+}\right)$and $642 \mathrm{keV}\left(6^{+} \rightarrow 4^{+}\right)$direct feeding transitions, recorded with the whole JUROGAM array, and the depopulating transitions recorded with the JUROGAM detectors at $158^{\circ}$ or $134^{\circ}$, in order to extract the lifetimes of the $2^{+}$and $4^{+}$states, respectively. In order to extract directly feeding $\gamma$-ray intensities, gates were set below the states of interest. Examples of recoil-gated $\gamma \gamma$-coincidence spectra are 


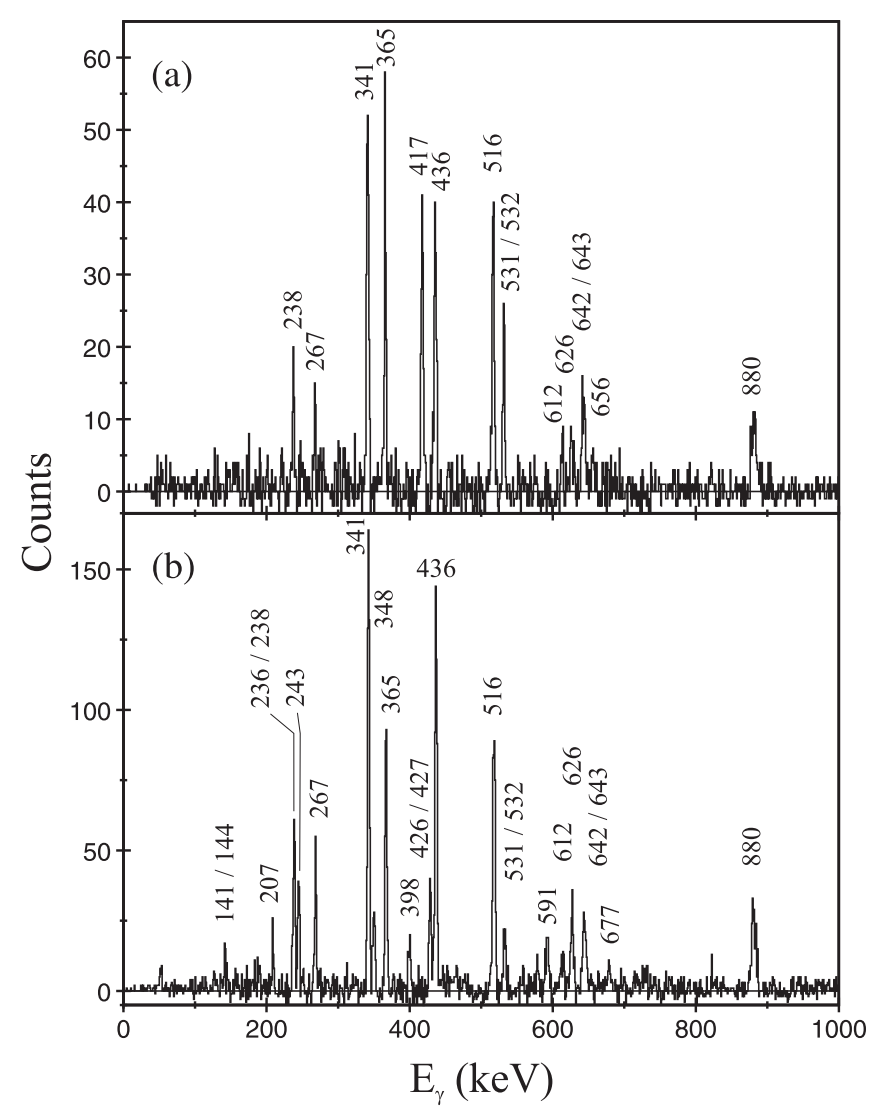

FIG. 4. Double-gated $\gamma$-ray coincidence spectra correlated with a recoil implantation in the GREAT spectrometer. Spectra providing evidence for a new decay path from the $\left(12^{+}\right)$state to the $9^{-}$state in Band 3. (a) Spectrum showing $\gamma$ rays in coincidence with the 243 and $532 \mathrm{keV}$ transitions that connect band 2 with band 3. (b) Spectrum showing $\gamma$ rays in coincidence with the $531 / 532 \mathrm{keV}$ and $417 \mathrm{keV}$ transitions.

shown in Fig. 6 and decay curves are shown in Figs. 7 and 8. The present variant of DDCM, in which the full line shape is used for gating, has been used and discussed in Refs. [27,29]. The standard DDCM procedure, in which gates are set on the fully shifted components of the transitions of interest was impossible in the present case due to the limited statistics in the $\gamma \gamma$-coincidence spectra.

The lower intensities of the higher-lying transitions precluded lifetime measurements using $\gamma \gamma$ coincidences. Instead these lifetimes were obtained using $\gamma$-ray singles data correlated with recoil implantations followed within $6.3 \mathrm{~s}$ by a characteristic $\alpha$ decay of ${ }^{168}$ Os [30] in the same DSSD pixel. The influence of the unobserved feeding transitions on the lifetime of interest cannot be eliminated when extracting lifetimes from singles $\gamma$-ray spectra, so it was assumed that the time behavior of the observed and unobserved feeding was similar [27,31].

The present work establishes that the 642 and $516 \mathrm{keV}$ transitions are self-coincident doublets; see Fig. 2. However, a significant influence of the $643 \mathrm{keV}$ transition on the extracted lifetimes can be ruled out based on the fact that weak degraded components were observed below these high-lying doublet transitions as shown in Fig. 9 for the $642 \mathrm{keV}$ transition.

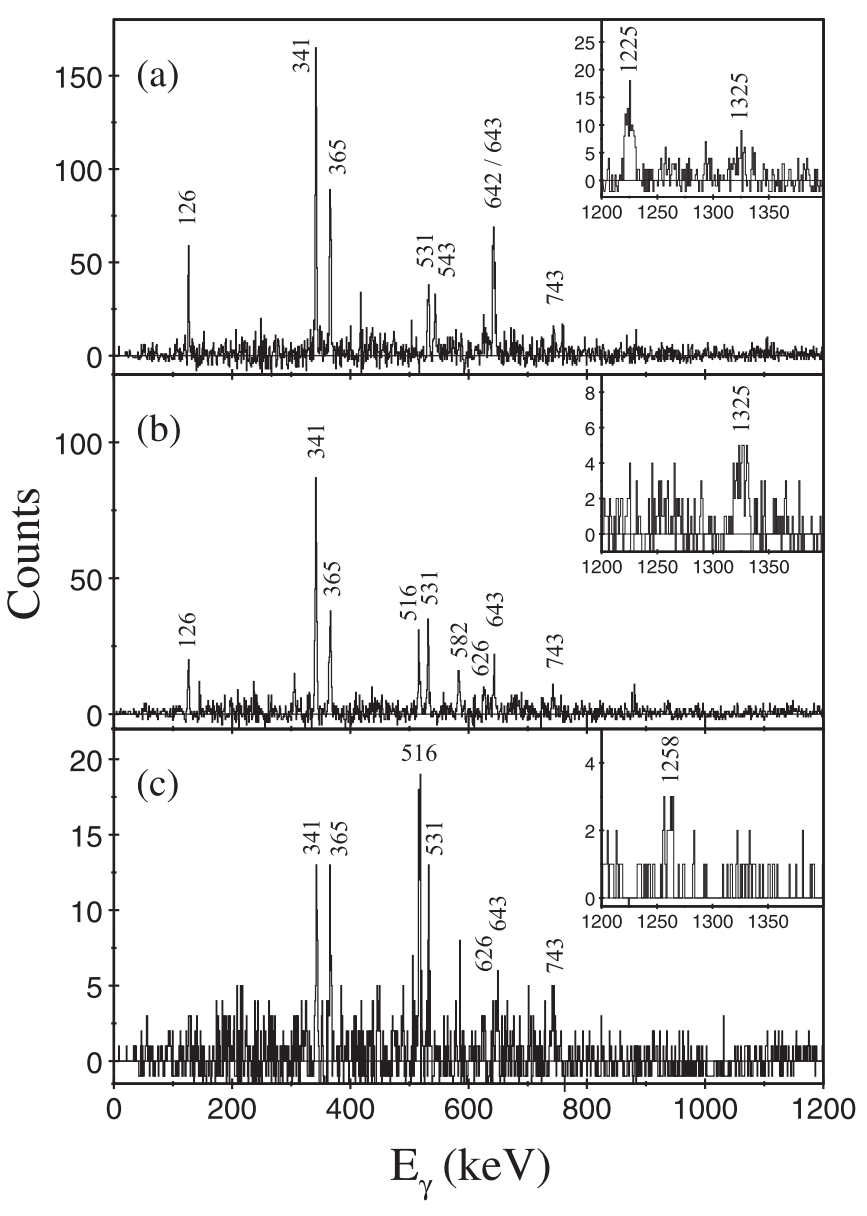

FIG. 5. Double-gated $\gamma$-ray coincidence spectra correlated with a recoil implantation in the GREAT spectrometer. Spectra show a new decay path from the $\left(12^{+}\right)$state to Band 1 . Insets to the figure show the high-energy linking transitions from Band 1 to the ground-state band. (a) Spectrum showing $\gamma$ rays in coincidence with both transitions of the $516 \mathrm{keV}$ doublet. (b) Spectrum showing $\gamma$ rays in coincidence with the 516 and $543 \mathrm{keV}$ transitions. (c) Spectrum showing $\gamma$ rays in coincidence with the 543 and $582 \mathrm{keV}$ transitions.

This implies that the $626 \mathrm{keV}$ transition and all the preceding ones in the cascade are fast compared to the transitions under investigation. As defined by Eq. (1), the feeding intensity is determined by the degraded component and therefore the time behavior of the doublet transitions do not interfere with the current DDCM analysis. Furthermore, the relative intensity of the $515.5 \mathrm{keV}$ transition (Table II) is less than $10 \%$ of that of $516.3 \mathrm{keV}$ transition and thus any influence falls within the statistical error bars of the extracted lifetime.

Table III lists the measured mean lifetimes $\tau$, reduced transition probabilities $B(E \lambda)$, and the absolute values for the transition dipole $\left(D_{\mathrm{t}}\right)$ and quadrupole $\left(Q_{\mathrm{t}}\right)$ moments.

\section{DISCUSSION}

\section{A. The excited positive-parity structure}

Band 1 is assigned to be a positive-parity structure that forms one of the decay paths from the $\left(12^{+}\right)$state in band 2. Similar decay paths to positive-parity bands have been 


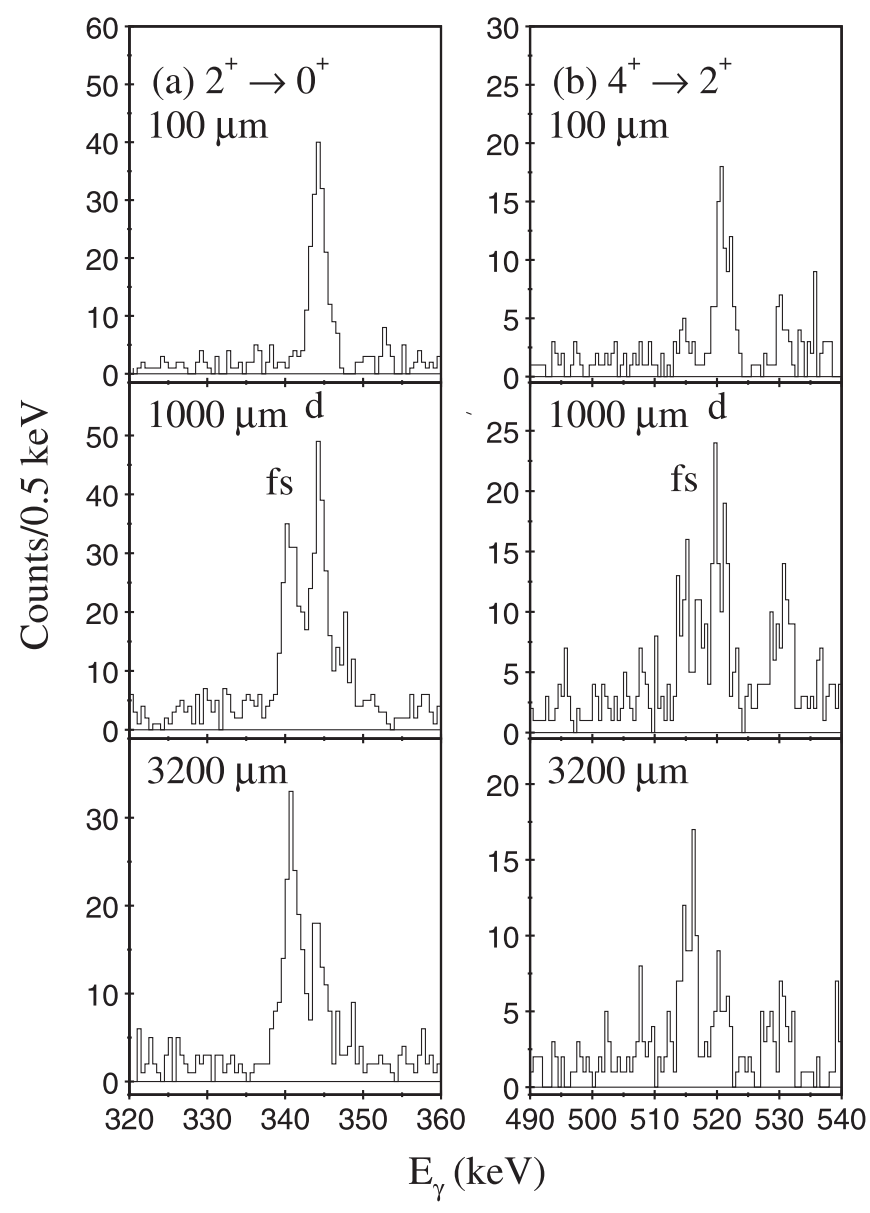

FIG. 6. Recoil-gated $\gamma \gamma$ coincidence spectra of ${ }^{168}$ Os recorded at three different target-to-degrader distances with five JUROGAM Ge detectors at $158^{\circ}$. (a) $\gamma$ rays in coincidence with the $516 \mathrm{keV}$ $4_{1}^{+} \rightarrow 2_{1}^{+}$transition. (b) $\gamma$ rays in coincidence with the $642 \mathrm{keV}$ $6_{1}^{+} \rightarrow 4_{1}^{+}$transitions. The fully Doppler-shifted (s) and degraded (d) components of the $341 \mathrm{keV}$ and $516 \mathrm{keV} \gamma$-ray transitions are labeled.

observed in nearby nuclei such as ${ }^{156}$ Dy [32] and ${ }^{158} \mathrm{Er}$ [33]. Excited positive-parity bands have been observed in the heavier even- $N$ Os isotopes than ${ }^{170}$ Os in experiments probing non-yrast states populated in the $\beta$ decay of the odd-odd Ir precursors [9,34]. Figure 10 compares band 1 in ${ }^{168} \mathrm{Os}$ with the systematic trends established for the heavier isotopes. These trends resemble those established for the $\mathrm{W}$ isotopes by Kibédi et al., who found that the states in the first excited positive-parity bands have a parabolic energy dependence on neutron number [35].

\section{B. Reduced transition probabilities and moments}

The measured intraband $B(E 2)$ values can be related to the transition quadrupole moment $Q_{\mathrm{t}}$ through the equation

$$
B\left(E 2 ; I_{\mathrm{i}} \rightarrow I_{\mathrm{f}}\right)=\frac{5}{16 \pi} e^{2} Q_{t}^{2}\left\langle I_{i} 020 \mid I_{f} 0\right\rangle^{2} .
$$

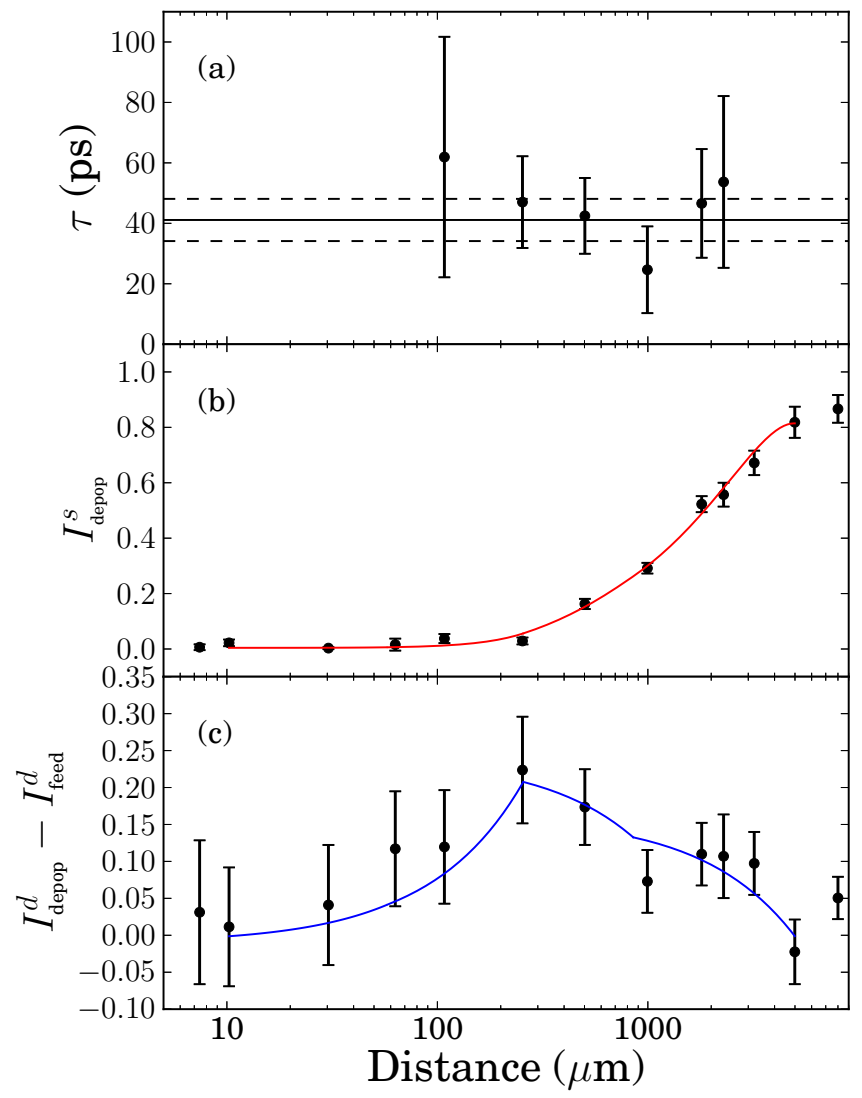

FIG. 7. Lifetime determination using the differential decay curve method (DDCM). (a) The individual mean lifetimes determined within the region of sensitivity [28] and the final mean lifetime and error bar as the solid and dashed horizontal lines, respectively. (b) The decay curve for the $2^{+}$state extracted from the coincidence spectra gated directly above the states under investigation and recorded with five $\mathrm{Ge}$ detectors at $158^{\circ}$. The line drawn through the experimental points is the fit of the decay curve. (c) The numerator of Eq. (1) and the derivative of the decay curve.

Similarly, the measured $B(E 1)$ values can be expressed in terms of the transition dipole moment $D_{t}$ with the relation

$$
B\left(E 1 ; I_{\mathrm{i}} \rightarrow I_{\mathrm{f}}\right)=\frac{3}{4 \pi} D_{t}^{2}\left\langle I_{i} 010 \mid I_{f} 0\right\rangle^{2} .
$$

Equations (2) and (3) hold for an axially symmetric rotating nucleus with $K=0$. Although these relations are not exact for the transitional case where nonaxial degrees of freedom are expected to occur, the quantities $\left|Q_{\mathrm{t}}\right|$ and $\left|D_{\mathrm{t}}\right|$ can be regarded as parameters in the context of the present work, in which they will be used for systematic comparisons. The $B(E 2) / B(E 1)$ branches for the negative-parity states have been extracted from the measured lifetimes using the relative $\gamma$-ray intensities given in Table II. The $B(E 1)$ transition probabilities for transitions from the $\left(7^{-}\right)$and $\left(9^{-}\right)$states in band 3 are large (see Table III) and support their interpretation as an octupole vibrational band at low frequency. 


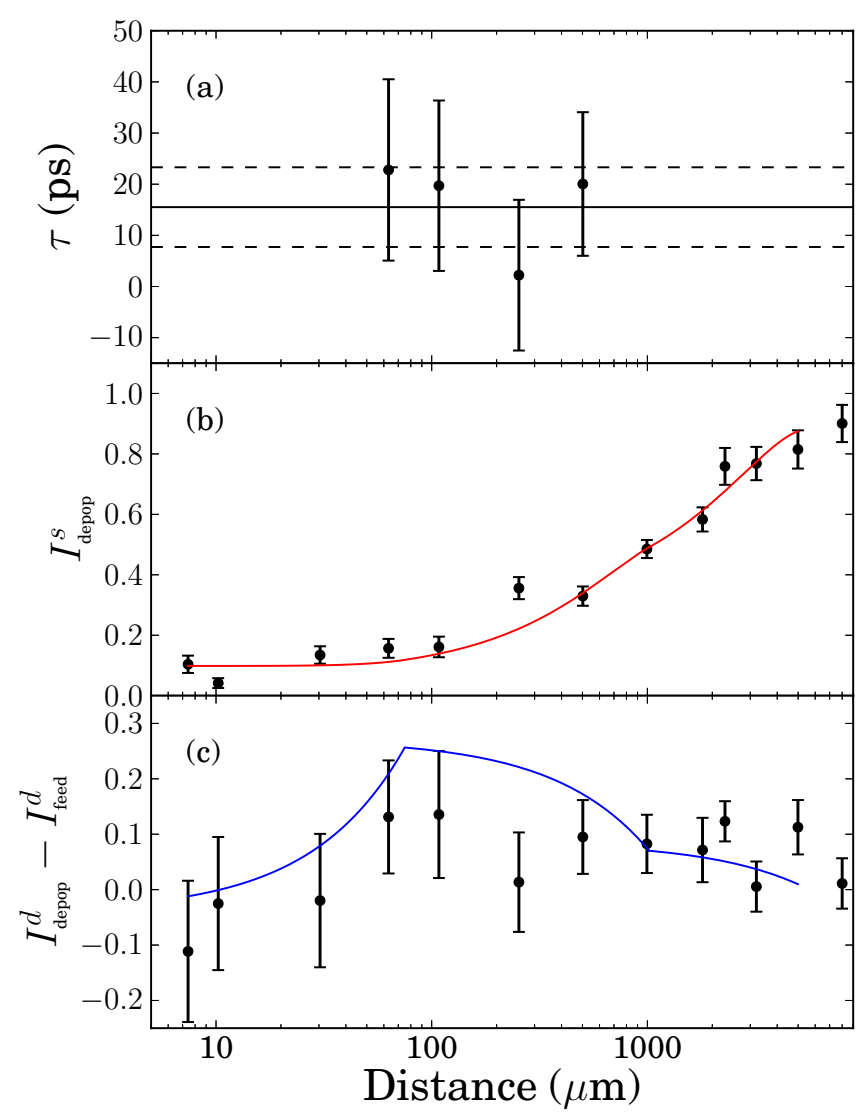

FIG. 8. Lifetime determination using the differential decay curve method (DDCM). (a) The individual mean lifetimes determined within the region of sensitivity [28] and the final mean lifetime and error bar as the solid and dashed horizontal lines, respectively. (b) The decay curve for the $4^{+}$state extracted from the coincidence spectra gated directly above the states under investigation and recorded with five Ge detectors at $158^{\circ}$. The line drawn through the experimental points is the fit of the decay curve. (c) The numerator of Eq. (1) and the derivative of the decay curve.

\section{The $B\left(E 2 ; 4_{1}^{+} \rightarrow 2_{1}^{+}\right) / B\left(E 2 ; 2_{1}^{+} \rightarrow 0_{1}^{+}\right)$ratio}

The smooth variation of the $E_{4 / 2}$ ratio between the vibrational and rotational regimes belies an intriguing feature

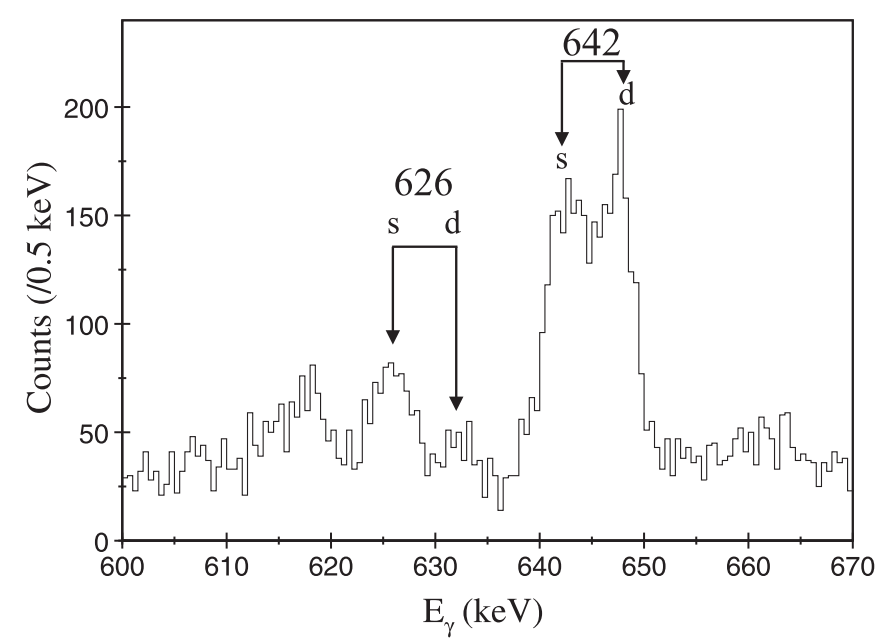

FIG. 9. Sum of $\gamma$-ray spectra at $158^{\circ}$ for all target-to-degrader distances. The $\gamma$-ray energies are Doppler corrected using the initial recoil velocity. The fully shifted (s) component of the $626 \mathrm{keV}$ transition is much stronger than its degraded (d) counterpart, which should lie at around $6 \mathrm{keV}$ higher in energy. In contrast, the fully shifted and degraded components of the $642 \mathrm{keV}$ peak have comparable intensities. This comparison indicates that the lifetime of the $626 \mathrm{keV}$ and its preceding transitions are shorter lived.

in the reduced transition probabilities of ${ }^{168} \mathrm{Os}_{92}$. Figure 1(b) shows the variation of the $B_{4 / 2}$ ratio as a function of neutron number for the Os isotopes. The measured ratios extracted for the $N \geqslant 96$ isotopes indicate values that are typical of rotational nuclei. The $B_{4 / 2}$ ratios reflect the tendency of $B(E 2)$ values to increase as a function of spin within a rotational band structure at low spin, as generally predicted in collective models [36]. The ratio deduced for ${ }^{168} \mathrm{Os}, B_{4 / 2}=0.34(18)$, shows a marked deviation from the heavier isotopes and seems to suggest a remarkably low collectivity of the $4_{1}^{+} \rightarrow 2_{1}^{+}$ transition compared with the $2_{1}^{+} \rightarrow 0_{1}^{+}$transition, see Fig. $1(\mathrm{c})$. In an extreme case if ${ }^{168}$ Os were an axially symmetric rotor, one would expect a $B_{4 / 2}=1.43$, which for the measured $2^{+}$lifetime would require a $4^{+}$state lifetime of $\tau \approx 3.5 \mathrm{ps}$.

This striking behavior was not anticipated in theoretical calculations for ${ }^{168}$ Os. Previous calculations include a

TABLE III. Electromagnetic properties of ${ }^{168}$ Os extracted from the present work.

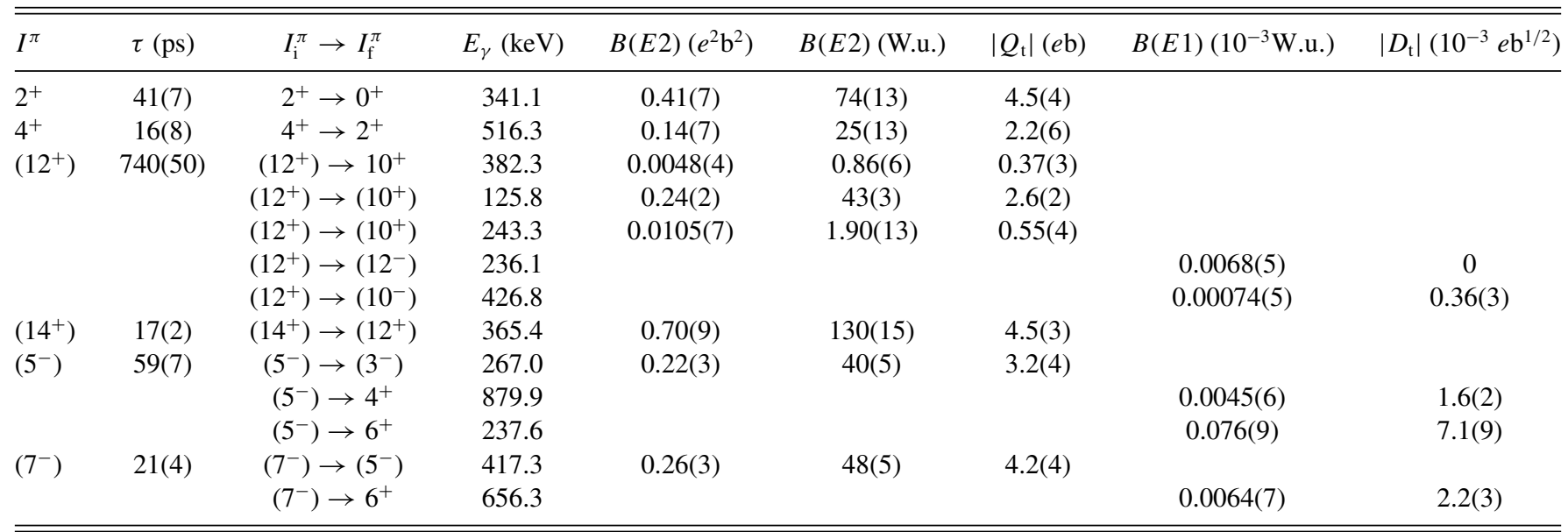




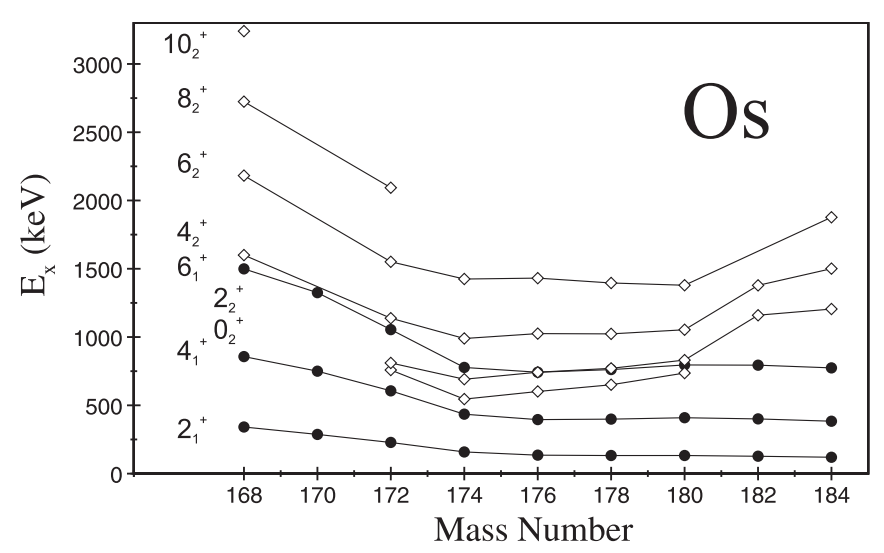

FIG. 10. Energy levels in the ground-state band (filled circles) and excited positive-parity structure (unfilled circles) as a function of mass number. The data are labeled by their assigned spins and parities. Data for the $A \geqslant 170$ Os isotopes are taken from Ref. [34].

QCD-inspired relativistic energy-density functional approach [37] in which systematic ground-state deformations across isotopic chains were compared with experimental values derived from $B(E 2)$ values [38]. No sudden change in deformation was predicted at $N=92$ in these calculations although a small discontinuity was present at $N=98$. Smoothly varying behavior was also predicted by other theoretical approaches including a number-projected BCS model [39] and a QRPA description based on realistic interactions [40].

In this work, spectroscopic calculations of the excited state energies and absolute $B(E 2)$ values have been performed within the proton-neutron interacting boson model (IBM-2) [41]. The parameters of IBM-2 were determined by mapping the potential energy surface, calculated by a constrained Hartree-Fock plus BCS (HF+BCS) calculation [42] with the Skyrme SkM* (or the "modified SkM") interaction [43], onto the classical limit of the IBM-2 Hamiltonian. Details of the calculation technique can be found in Refs. [44,45]. The proton and neutron effective charges were set to be equal and fixed at $e_{\pi}=e_{\nu}=0.12 \mathrm{eb}$ by the comparison between the intrinsic quadrupole moments obtained from the HF+BCS calculation and the IBM-2 model. It should be noted that the following result does not depend on the choice of the interaction at the qualitative level, as the topology of the potential energy surface around the global minimum remains unchanged. This is confirmed by the fact that both the Skyrme SkM* and the SLy4 interactions predict the same $\beta_{2}$ deformation value on the prolate axis and similar collective patterns are predicted by the mapped IBM. The comparison between the hybrid $\mathrm{SkM}^{*}$-IBM and the experimental data is shown in Fig. 11. The energy levels are reasonably well reproduced in the groundstate band but the theoretical calculations predict $B(E 2)$ values that follow the characteristic trend of a rotational band that do not agree with the measurements.

There are two known circumstances in which a sudden loss of collectivity in a ground-state band can occur. The first is in a seniority dominated structure where the $B\left(E 2 ; 2_{1}^{+} \rightarrow\right.$ $\left.0_{1}^{+}\right)$strength reflects a $v=2 \rightarrow v=0 E 2$ transition and the $B\left(E 2 ; 4_{1}^{+} \rightarrow 2_{1}^{+}\right)$strength reflects a $v=2 \rightarrow v=2 E 2$ transition [46] where $v$ is the seniority quantum number.

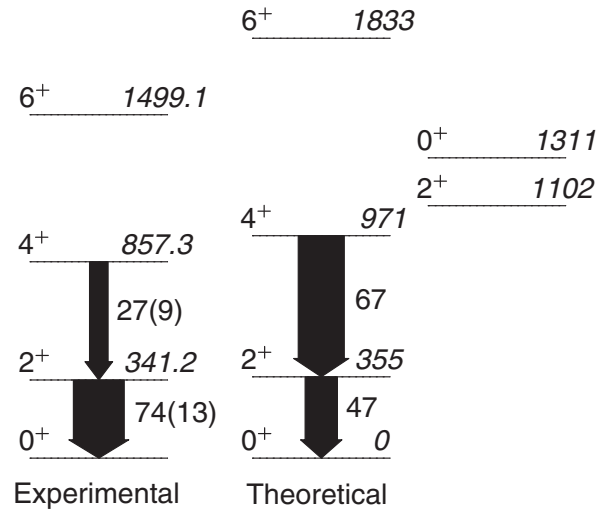

FIG. 11. Comparison of experimental and theoretical results of the present study. The level energies are given in $\mathrm{keV}$. The width of the arrows are proportional to the $B(E 2)$ values, which are given next to the arrows in W.u.

Generally, seniority structures are only known to occur at and near to closed shells, and possibly at subshells $[47,48]$. This mechanism is thought to be unlikely for the anomaly in ${ }^{168} \mathrm{Os}$ since its $N$ and $Z$ are rather far from magicity and subshell gaps are not expected.

The second scenario for a lower collectivity arises from shape coexistence where the $B\left(E 2 ; 2_{1}^{+} \rightarrow 0_{1}^{+}\right)$strength reflects an intra-band $E 2$ transition and the $B\left(E 2 ; 4_{1}^{+} \rightarrow 2_{1}^{+}\right)$strength reflects an interband $E 2$ transition as observed in nuclei such as ${ }^{184} \mathrm{Hg}$ [49]. The main reservation for attributing the anomaly to coexisting intruder structures is that such features generally only appear in regions near to closed shells for protons and near midshell (or near a subshell) for the neutrons or vice versa [3]. Furthermore, band-mixing calculations for ${ }^{168}$ Os suggest that the deformed intruder band head in ${ }^{168} \mathrm{Os}$ lies at an excitation energy around $1.8 \mathrm{MeV}$ [7].

The excited positive-parity structure (band 1) observed in this work might provide an alternative candidate for mixing with the yrast states. Figure 11 shows the IBM-2 predictions for the excitation energies of the $\beta\left(K^{\pi}=0^{+}\right)$and $\gamma\left(K^{\pi}=2^{+}\right)$ band heads at 1311 and $1102 \mathrm{keV}$, respectively. The predicted $\mathrm{O}_{2}^{+}$state lies at an energy consistent with extrapolations of the measured energy level systematics shown in Fig. 10. The calculations suggest that mixing with the non-yrast states is not sufficient to account for the anomalous $B_{4 / 2}$ ratios. Thus, it seems unlikely that the anomalous $B(E 2)$ values arise from mixing with coexisting states. However ${ }^{168} \mathrm{Os}$ has a $E_{4 / 2}$ ratio that is consistent with that expected for a transitional $\gamma$-soft nucleus, see Fig. 1(a), so shape fluctuations may give rise to significant mixing but calculating this is beyond the scope of this work.

\section{CONCLUSIONS}

The level scheme for ${ }^{168}$ Os has been extended in a $\gamma$-ray coincidence experiment using the JUROGAM spectrometer used in conjunction with the RITU gas-filled separator and the GREAT focal plane spectrometer. Recoil distance Dopplershift lifetime measurements using the differential decay curve method have been performed in a complementary experiment using these devices in conjunction with the IKP Köln plunger. 
The particularly small $B\left(E 2 ; 4_{1}^{+} \rightarrow 2_{1}^{+}\right) / B\left(E 2 ; 2_{1}^{+} \rightarrow 0_{1}^{+}\right)$ ratio deduced for ${ }^{168}$ Os could not be reproduced using IBM-2 model calculations based on the SkM* energy-density functional. This anomaly appears unlikely to arise from seniority or shape coexistence phenomena. Further work is required to investigate whether the expected triaxiality of ${ }^{168} \mathrm{Os}$ might contribute to this unusual observation.

\section{ACKNOWLEDGMENTS}

This work has been supported through EURONS (European Commission Contract No. RII3-CT-2004-506065), the
Academy of Finland under the Finnish Centre of Excellence Programme 2006-2011 (Nuclear and Accelerator Based Physics Contract No. 213503), and the UK Science and Technology Facilities Council. The UK/France (STFC/IN2P3) Loan Pool and GAMMAPOOL network are acknowledged for the EUROGAM detectors of JUROGAM. T.G., P.T.G., and C.S. acknowledge the support of the Academy of Finland, Contracts No. 131665, No. 111965, and No. 209430, respectively. Financial support (K.H.) from the "FWO-Vlaanderen" and the InterUniversity Attraction Pole Programme, Belgian State Federal Office for Scientific, Technical and Cultural Affairs (IAP Grant No. P7/12) is acknowledged.
[1] A. Bohr and B. R. Mottelson, Nuclear Structure Volume 1: Single-Particle Motion (W.A. Benjamin, New York, 1969).

[2] A. Bohr and B. R. Mottelson, Nuclear Structure Volume 2: Nuclear Deformation (W.A. Benjamin, New York, 1975).

[3] K. Heyde and J. L. Wood, Rev. Mod. Phys. 83, 1467 (2011).

[4] D. T. Joss, K. Lagergren, D. E. Appelbe, C. J. Barton, J. Simpson, B. Cederwall, B. Hadinia, R. Wyss, S. Eeckhaudt, T. Grahn, P. T. Greenlees, P. M. Jones, R. Julin, S. Juutinen, H. Kettunen, M. Leino, A.-P. Leppänen, P. Nieminen, J. Pakarinen, P. Rahkila, C. Scholey, J. Uusitalo, R. D. Page, E. S. Paul, and D. R. Wiseman, Phys. Rev. C 70, 017302 (2004).

[5] Z. Podolyak et al., Phys. Rev. C 79, 031305(R) (2009).

[6] M. C. Drummond, D. T. Joss, R. D. Page, J. Simpson, D. O’Donnell, K. Andgren, L. Bianco, B. Cederwall, I. G. Darby, S. Eeckhaudt, M. B. Gomez-Hornillos, T. Grahn, P. T. Greenlees, B. Hadinia, P. M. Jones, R. Julin, S. Juutinen, S. Ketelhut, A. P. Leppanen, M. Leino, M. Nyman, J. Pakarinen, P. Rahkila, M. Sandzelius, P. J. Sapple, J. Saren, B. Saygi, C. Scholey, J. Sorri, J. Thomson, J. Uusitalo, and M. Venhart, Phys. Rev. C 87, 054309 (2013).

[7] D. T. Joss et al., Nucl. Phys. A 689, 631 (2001).

[8] J. L. Durell, G. D. Dracoulis, C. Fahlander, and A. P. Byrne, Phys. Lett. B 115, 367 (1982).

[9] P. M. Davidson, G. D. Dracoulis, T. Kibedi, A. P. Byrne, S. S. Anderssen, A. M. Baxter, B. Fabricius, G. J. Lane, and A. E. Stuchbery, Nucl. Phys. A 568, 90 (1994).

[10] C. Wheldon et al., Nucl. Phys. A 699, 415 (2002).

[11] P. D. Bond, R. F. Casten, D. D. Warner, and D. Horn, Phys. Lett. B 130, 167 (1983).

[12] A. de Shalit and I. Talmi, Nuclear Shell Theory (Academic, New York, 1963).

[13] R. B. Cakirli, R. F. Casten, J. Jolie, and N. Warr, Phys. Rev. C 70, 047302 (2004).

[14] E. Williams, C. Plettner, E. A. McCutchan, H. Levine, N. V. Zamfir, R. B. Cakirli, R. F. Casten, H. Ai, C. W. Beausang, G. Gürdal, A. Heinz, J. Qian, D. A. Meyer, N. Pietralla, and V. Werner, Phys. Rev. C 74, 024302 (2006).

[15] G. de Angelis et al., Phys. Lett. B 535, 93 (2002).

[16] O. Moller, N. Warr, J. Jolie, A. Dewald, A. Fitzler, A. Linnemann, K. O. Zell, P. E. Garrett, and S. W. Yates, Phys. Rev. C 71, 064324 (2005).

[17] C. Louchart et al., Phys. Rev. C 87, 054302 (2013).

[18] D. Hertz-Kintish, L. Zamick, and S. J. Q. Robinson, Phys. Rev. C 90, 034307 (2014).
[19] Data extracted using the NNDC On-Line Data Service from the ENSDF database, file revised as of 22 Feb 2010. M. R. Bhat, Evaluated Nuclear Structure Data File (ENSDF), Nuclear Data for Science and Technology, edited by S. M. Qaim (SpringerVerlag, Berlin, 1992), p. 817.

[20] C. W. Beausang et al., Nucl. Instrum. Methods Phys. Res., Sect. A 313, 37 (1992).

[21] M. Leino et al., Nucl. Instrum. Methods Phys. Res., Sect. B 99, 653 (1995)

[22] R. D. Page et al., Nucl. Instrum. Methods Phys. Res., Sect. B 204, 634 (2003).

[23] I. H. Lazarus et al., IEEE Trans. Nucl. Sci. 48, 567 (2001).

[24] P. Rahkila, Nucl. Instrum. Methods Phys. Res., Sect. A 595, 637 (2008)

[25] D. C. Radford, Nucl. Instrum. Methods Phys. Res., Sect. A 361, 297 (1995).

[26] L. Cleemann et al., Nucl. Instrum. Methods 156, 477 (1978).

[27] T. Grahn, A. Petts, M. Scheck, P. A. Butler, A. Dewald, M. B. Gomez-Hornillos, P. T. Greenlees, A. Gorgen, K. Helariutta, J. Jolie, P. Jones, R. Julin, S. Juutinen, S. Ketelhut, R. Krucken, T. Kroll, M. Leino, J. Ljungvall, P. Maierbeck, B. Melon, M. Nyman, R. D. Page, T. Pissulla, P. Rahkila, J. Saren, C. Scholey, A. Semchenkov, J. Sorri, J. Uusitalo, R. Wadsworth, and M. Zielinska, Phys. Rev. C 80, 014324 (2009).

[28] A. Dewald, S. Harissopulos, and P. von Brentano, Z. Phys. A 334, 163 (1989).

[29] A. Dewald, R. Peusquens, B. Saha, P. von Brentano, A. Fitzler, T. Klug, I. Wiedenhöver, M. P. Carpenter, A. Heinz, R. V. F. Janssens, F. G. Kondev, C. J. Lister, D. Seweryniak, K. A. Saleem, R. Krücken, J. R. Cooper, C. J. Barton, K. Zyromski, C. W. Beausang, Z. Wang, P. Petkov, A. M. Oros-Peusquens, U. Garg, and S. Zhu, Phys. Rev. C 68, 034314 (2003).

[30] R. D. Page, P. J. Woods, R. A. Cunningham, T. Davinson, N. J. Davis, A. N. James, K. Livingston, P. J. Sellin, and A. C. Shotter, Phys. Rev. C 53, 660 (1996).

[31] M. Scheck, T. Grahn, A. Petts, P. A. Butler, A. Dewald, L. P. Gaffney, M. B. Gomez-Hornillos, A. Görgen, P. T. Greenlees, K. Helariutta et al., Phys. Rev. C 81, 014310 (2010).

[32] M. A. Riley et al., Nucl. Phys. A 486, 456 (1988).

[33] T. S. Dinoko et al., EPJ Web of Conferences 63, 01005 (2013).

[34] T. Kibedi, G. D. Dracoulis, A. P. Byrne, and P. M. Davidson, Nucl. Phys. A 567, 183 (1994).

[35] T. Kibedi, G. D. Dracoulis, A. P. Byrne, and P. M. Davidson, Nucl. Phys. A 688, 669 (2001). 
[36] D. J. Rowe and J. L. Wood, Fundamentals of Nuclear Models: Foundational Models (World Scientific, Singapore, 2009).

[37] P. Finelli, N. Kaiser, D. Vretenar, and W. Weise, Nucl. Phys. A 770, 1 (2006).

[38] S. Raman, C. W. Nestor, and P. Tikkanen, At. Data Nucl. Data Tables 78, 1 (2001).

[39] N. Benhamouda, N. H. Allal, M. Fellah, and M. R. Oudih, in Proceedings of the 13th International Symposium on Capture Gamma-Ray Spectroscopy and Related Topics, August 2008, Cologne, edited by J. Jolie, A. Zilges, N. Warr, and A. Blazhev AIP Conf. Proc. No. 1090 (AIP, New York, 2009), p. 613.

[40] S. Peltonen, D. Delion, and J. Suhonen, Phys. Rev. C 71, 044315 (2005).

[41] T. Otsuka et al., Nucl. Phys. A 309, 1 (1978).

[42] P. Bonche et al., Comput. Phys. Commun. 171, 49 (2005).
[43] J. Bartel et al., Nucl. Phys. A 386, 79 (1982).

[44] K. Nomura, N. Shimizu, and T. Otsuka, Phys. Rev. Lett. 101, 142501 (2008).

[45] K. Nomura, N. Shimizu, and T. Otsuka, Phys. Rev. C 81, 044307 (2010)

[46] J. J. Ressler, R. F. Casten, N. V. Zamfir, C. W. Beausang, R. B. Cakirli, H. Ai, H. Amro, M. A. Caprio, A. A. Hecht, A. Heinz, S. D. Langdown, E. A. McCutchan, D. A. Meyer, C. Plettner, P. H. Regan, M. J. S. Sciacchitano, and A. D. Yamamoto, Phys. Rev. C 69, 034317 (2004).

[47] P. Van Isacker and S. Heinze, Phys. Rev. Lett. 100, 052501 (2008).

[48] K. Sieja, F. Nowacki, K. Langanke, and G. Martínez-Pinedo, Phys. Rev. C 79, 064310 (2009).

[49] W. C. Ma et al., Phys. Lett. B 167, 277 (1986). 